QUARTERLY OF APPLIED MATHEMATICS

VOLUME LXIV, NUMBER 3

SEPTEMBER 2006, PAGES 499-513

$\mathrm{S}$ 0033-569X(06)01010-4

Article electronically published on April 6, 2006

\title{
EXPONENTIAL STABILITY IN LINEAR VISCOELASTICITY
}

BY

\author{
VITTORINO PATA
}

Dipartimento di Matematica "F.Brioschi", Politecnico di Milano, Via Bonardi 9, 20133 Milano, Italy

\begin{abstract}
We address the study of the asymptotic behavior of solutions to an abstract integrodifferential equation modeling linear viscoelasticity. Framing the equation in the past history setting, we analyze the exponential stability of the related semigroup $S(t)$ with dependence on the convolution kernel, providing a more general sufficient condition than the usual one present in the literature.
\end{abstract}

1. Introduction. Given a Hilbert space $(H,\langle\cdot, \cdot\rangle,\|\cdot\|)$ and a strictly positive selfadjoint linear operator $A: \mathcal{D}(A) \subset H \rightarrow H$, we consider, for $t>0$, the second-order linear integrodifferential equation

$$
\partial_{t t} u(t)+\alpha A u(t)+\beta \partial_{t} u(t)-\int_{0}^{\infty} \mu(s) A u(t-s) d s=0,
$$

where $\alpha>0, \beta \geq 0$ and the convolution (or memory) kernel $\mu$ is a summable decreasing function defined on $\mathbb{R}^{+}=(0, \infty)$, hence nonnegative, of total mass

$$
\kappa=\int_{0}^{\infty} \mu(s) d s \in(0, \alpha)
$$

Equation (1.1), supplemented by the initial conditions

$$
\left\{\begin{array}{l}
u(t)=w_{0}(t), \quad t \leq 0, \\
u_{t}(0)=v_{0},
\end{array}\right.
$$

with $w_{0}$ and $v_{0}$ assigned data, serves as a model to describe the dynamics of linearly viscoelastic solids. In that case, $A$ is the negative Laplacian with Dirichlet boundary conditions acting on $L^{2}(\Omega)$, where $\Omega$ is the volume occupied by the viscoelastic body at rest, and $u$ represents the displacement field relative to the reference configuration. The term $\beta \partial_{t} u$ accounts for dynamical friction.

Introducing the Hilbert space $V=\mathcal{D}\left(A^{1 / 2}\right)$ continuously embedded into $H$ and the $L^{2}$-weighted space $\mathcal{M}=L_{\mu}^{2}\left(\mathbb{R}^{+} ; V\right)$, both endowed with their natural inner products,

Received November 14, 2005.

2000 Mathematics Subject Classification. Primary 35B40, 45K05, 45M10, 47D06.

Key words and phrases. Linear viscoelasticity, memory kernels, contraction semigroups, exponential stability.

E-mail address: pata@mate.polimi.it 
along with the linear operator $T=-\partial_{s}$ on $\mathcal{M}$ (derivative in the distributional sense) defined on the domain

$$
\mathcal{D}(T)=\left\{\eta \in \mathcal{M}: \partial_{s} \eta \in \mathcal{M}, \lim _{s \rightarrow 0} \eta(s)=0\right\},
$$

we can rephrase equation (1.1) as an abstract system of PDEs on the Hilbert space $\mathcal{H}=H \times V \times \mathcal{M}$. Precisely,

$$
\left\{\begin{array}{l}
\partial_{t t} u(t)+\kappa \omega A u(t)+\beta \partial_{t} u(t)+\int_{0}^{\infty} \mu(s) A \eta^{t}(s) d s=0, \\
\partial_{t} \eta^{t}=T \eta^{t}+\partial_{t} u(t)
\end{array}\right.
$$

having put

$$
\omega=\frac{\alpha-\kappa}{\kappa} .
$$

This is the so-called past history setting of (1.1), for it is obtained from (1.1), following a brilliant intuition of Dafermos [4, 5], by introducing the supplementary variable

$$
\eta^{t}(s)=u(t)-u(t-s), \quad t \geq 0, s>0,
$$

which reproduces the past history of $u$. Accordingly, the initial conditions for (1.2) read

$$
\left\{\begin{array}{l}
u(0)=u_{0} \\
u_{t}(0)=u_{1} \\
\eta^{0}(s)=\eta_{0}(s)
\end{array}\right.
$$

where $u_{0}=w_{0}(0)$ and $\eta_{0}(s)=w_{0}(0)-w_{0}(-s)$. We refer the reader to [12] for a detailed presentation of the historical approach to treating integrodifferential equations of this kind. The remarkable fact about this latter formulation is that, under quite general assumptions on the kernel $\mu$, system (1.2) generates a linear contraction semigroup $S(t)$ on $\mathcal{H}$.

The main question now is the following:

$$
\text { Is } S(t) \text { exponentially stable? }
$$

This amounts to finding constants $M \geq 1$ and $\varepsilon>0$ such that

$$
\|S(t)\|_{L(\mathcal{H})} \leq M e^{-\varepsilon t},
$$

where $L(\mathcal{H})$ is the Banach space of bounded linear operators on $\mathcal{H}$. This was indeed the main focus of several works that have appeared over the years (e.g., 7, 8, 10, 11, 13, [14, 16]). The situation is of course more delicate for the case $\beta=0$, where the entire dissipation is given solely by the memory integral. To the best of our knowledge, all positive results regarding exponential stability of semigroups arising from problems with memory have been obtained assuming the following condition on $\mu$ (cf. [14]):

$$
\mu^{\prime}(s)+\delta \mu(s) \leq 0,
$$

for some $\delta>0$ and (almost) every $s \in \mathbb{R}^{+}$. In fact, if (1.3) holds, then our semigroup $S(t)$ is exponentially stable, even when $\beta=0$. This result has been proved first via Laplace transform methods [7], semigroup techniques [13, and direct energy estimates [1] (in the latter case, with some restrictions on the behavior of $\mu$ in a neighborhood 
of zero). Although it includes many physically interesting kernels, condition (1.3) is somehow unsatisfactory and certainly too restrictive. For instance, it does not allow $\mu$ to have flat zones, or even horizontal inflection points, when it should be conceivably true that exponential stability should be conserved if, say, we consider a kernel which is equal to the negative exponential, except on a small set. The recent paper [2] proves that a necessary condition for $S(t)$ to be exponentially stable is that there exist $C \geq 1$ and $\delta>0$ such that

$$
\mu(t+s) \leq C e^{-\delta t} \mu(s),
$$

for every $t \geq 0$ and almost every $s>0$, which is the same as requiring that

$$
\limsup _{t \rightarrow \infty}\left[\sup _{s \in(0, S)} \frac{\mu(t+s)}{\mu(s)}\right]<1
$$

where

$$
S=\sup \left\{s \in \mathbb{R}^{+}: \mu(s)>0\right\} .
$$

If $C=1$, then (1.4) is equivalent to (1.3). Nonetheless, the gap between the necessary condition (1.4) and the sufficient condition (1.3) is huge. For example, any compactly supported kernel $\mu$ fulfills (1.4), but it clearly need not satisfy (1.3).

The aim of the present work is to provide a much weaker sufficient condition in order for exponential stability to occur. Loosely speaking, our main result reads as follow.

Theorem 1.1. Assume that the necessary condition (1.4) holds. If $\beta=0$, then the semigroup $S(t)$ is exponentially stable, provided that the set where the kernel $\mu$ is flat is sufficiently small, with respect to the measure $\mu(s) d s$. If $\beta>0$, then (1.4) is sufficient as well in order to have exponential stability.

We now briefly sketch the plan of the paper. In $\S 2$ we specify the assumptions on the memory kernel $\mu$, and we recall the construction of the semigroup $S(t)$. In $\S 3$ we reformulate Theorem 1.1 in a precise way, while $\S 4, \S 5$, and $\S 6$ are devoted to the proof of the main result. Finally, in $\S 7$ we discuss further possible applications.

\section{The contraction semigroup $S(t)$.}

Assumptions on $\boldsymbol{\mu}$. Let $\mu$ be a monotone (possibly not strictly) decreasing summable function on $\mathbb{R}^{+}$of total mass $\kappa \in(0, \alpha)$. Setting $s_{0}=0$, we suppose that there exists a strictly increasing sequence $\left\{s_{n}\right\}$ (possibly finite, or even reduced solely to $s_{0}$ ) converging to $s_{\infty} \in[0, \infty]$ such that, for all $n \in \mathbb{N}, \mu$ has jumps at the point $s=s_{n}$, and it is absolutely continuous on each interval $I_{n}=\left(s_{n-1}, s_{n}\right)$ and on the interval $I_{\infty}=\left(s_{\infty}, \infty\right)$, unless $I_{\infty}$ is empty. If $s_{\infty}<\infty$, then $\mu$ may or may not have a jump at $s=s_{\infty}$. Under these conditions, $\mu^{\prime}$ is defined and is nonpositive almost everywhere. Finally, for $n \in \mathbb{N}$, we denote

$$
\mu_{n}=\mu\left(s_{n}^{-}\right)-\mu\left(s_{n}^{+}\right) \quad \text { and } \quad \mu_{\infty}=\mu\left(s_{\infty}^{-}\right)-\mu\left(s_{\infty}^{+}\right),
$$

with $\mu_{\infty}$ defined only if $s_{\infty}<\infty$. Note that $\mu$ can be (weakly) singular in a neighborhood of zero. 
As shown in 22, in the above assumptions, system (1.2) generates a contraction semigroup $S(t)=e^{t \mathbb{L}}$ on the phase space $\mathcal{H}=V \times H \times \mathcal{M}$, normed by

$$
\|(u, v, \eta)\|_{\mathcal{H}}^{2}=\kappa \omega\|u\|_{V}^{2}+\|v\|^{2}+\|\eta\|_{\mathcal{M}}^{2}
$$

The domain of the infinitesimal generator $\mathbb{L}$ of $S(t)$ is given by

$$
\mathcal{D}(\mathbb{L})=\left\{(u, v, \eta) \in \mathcal{H}: u+\int_{0}^{\infty} \mu(s) \eta(s) d s \in \mathcal{D}(A), v \in V, \eta \in \mathcal{D}(T)\right\} .
$$

In particular, the third component of the solution $S(t)\left(u_{0}, v_{0}, \eta_{0}\right)=\left(u(t), \partial_{t} u(t), \eta^{t}\right)$ has the explicit representation formula (see [16])

$$
\eta^{t}(s)= \begin{cases}u(t)-u(t-s), & 0<s \leq t, \\ \eta_{0}(s-t)+u(t)-u_{0}, & s>t .\end{cases}
$$

For $\eta \in \mathcal{D}(T)$, we introduce the quantity

$$
\mathbb{J}[\eta]=\sum_{n} \mu_{n}\left\|\eta\left(s_{n}\right)\right\|_{V}^{2},
$$

where the sum includes the value $n=\infty$ if $s_{\infty}<\infty$. Then, if $\left(u_{0}, v_{0}, \eta_{0}\right) \in \mathcal{D}(\mathbb{L})$, the energy equality in $\mathcal{H}$ reads (see [2, 12])

$$
\frac{d}{d t}\left\|\left(u(t), \partial_{t} u(t), \eta^{t}\right)\right\|_{\mathcal{H}}^{2}+2 \beta\left\|\partial_{t} u(t)\right\|^{2}+\int_{0}^{\infty}-\mu^{\prime}(s)\left\|\eta^{t}(s)\right\|_{V}^{2} d s+\mathbb{J}\left[\eta^{t}\right]=0 .
$$

3. The main theorem. In order to state the result, we have to introduce some definitions. Concerning the kernel $\mu$, in light of our purposes we give the following

DeFinition 3.1. We say that $\mu$ is an admissible kernel if it satisfies the above general assumptions and (1.4) holds.

We construct the probability measure $\hat{\mu}$ on $\mathbb{R}^{+}$as

$$
\hat{\mu}(\mathcal{A})=\frac{1}{\kappa} \int_{\mathcal{A}} \mu(s) d s,
$$

for every measurable set $\mathcal{A} \subset \mathbb{R}^{+}$.

Definition 3.2. We define the flatness set of the kernel $\mu$ as

$$
\mathcal{F}_{\mu}=\left\{s \in \mathbb{R}^{+}: \mu(s)>0 \text { and } \mu^{\prime}(s)=0\right\},
$$

and we call the quantity

$$
\mathcal{R}_{\mu}=\hat{\mu}\left(\mathcal{F}_{\mu}\right)
$$

the flatness rate of $\mu$.

Setting now, for $\omega>0$,

$$
M(\omega)= \begin{cases}\frac{\omega^{1 / 2}}{2}, & \omega<1 \\ \frac{1}{2}, & \omega \geq 1\end{cases}
$$

we are in a position to state

Theorem 3.3. Let $\mu$ be an admissible kernel. If $\beta=0$ and

$$
\mathcal{R}_{\mu}<M(\omega),
$$

then $S(t)$ is exponentially stable. If $\beta>0$, then $S(t)$ is always exponentially stable. 
A particular instance, albeit quite interesting in view of concrete applications, is the following

Corollary 3.4. Let $\mu$ be an admissible kernel. If $\mu^{\prime}<0$ almost everywhere on the support of $\mu$, then $S(t)$ is exponentially stable.

Theorem 3.3 provides an optimal result for the case $\beta>0$, whereas, for $\beta=0$, it locates a threshold for the flatness rate $\mathcal{R}_{\mu}$, above which we cannot say anything. Roughly speaking, if $\omega$ is large enough, we have a positive answer only if the measure of the flatness set does not exceed the measure of the set where $\mu$ is decreasing. Unfortunately, this threshold seems to be sharp, at least with respect to our techniques. Clearly, it would be interesting to say something for the case not covered by the theorem.

REMARK 3.5. The paper [2] shows that, if $\beta=0$ and $\mathcal{R}_{\mu}=1$ (i.e., $\mu$ is a step function), then there are particular forms of $\mu$ which allow the existence of periodic (hence, purely elastic) solutions. If in addition $H$ is a separable infinite-dimensional Hilbert space and $A$ has compact inverse (as in the case of linear viscoelasticity), then exponential stability never occurs, at least for a wide class of operators $A$, including the negative Laplacian on certain domains.

Thus, the question is really what happens when $\mathcal{R}_{\mu} \in[M(\omega), 1)$. We do not have a sensible conjecture (and there is no satisfactory numerical evidence either), so we leave it as an open question.

4. Some preliminary results. The proof of Theorem 3.3 is based on sharp estimates of certain auxiliary functionals defined for $\left(u_{0}, v_{0}, \eta_{0}\right) \in \mathcal{D}(\mathbb{L})$. We first need to establish some notation.

- We recall the Poincaré inequality, namely,

$$
\|w\|^{2} \leq \frac{1}{\lambda}\|w\|_{V}^{2}, \quad \forall w \in V
$$

where $\lambda>0$ is the infimum of the spectrum of $A$.

- Given a measurable set $\mathcal{F} \subset \mathbb{R}^{+}$, we consider the $L^{2}$-weighted space $\mathcal{M}_{\mathcal{F}}=L_{\mu}^{2}(\mathcal{F} ; V)$.

- We introduce the new variable

$$
\xi^{t}(s)=\eta^{t}(s)-u(t) .
$$

Note that, in view of the representation formula (2.1), it holds that

$$
\xi^{t}(s)= \begin{cases}-u(t-s), & 0<s \leq t, \\ \eta_{0}(s-t)-u_{0}, & s>t .\end{cases}
$$

- For any fixed $s_{*} \geq 0$, let $\psi=\psi_{s_{*}}: \mathbb{R}^{+} \rightarrow[0, \infty)$ be defined as

$$
\psi(s)=\mu\left(s_{*}\right) \chi_{\left(0, s_{*}\right]}(s)+\mu(s) \chi_{\left(s_{*}, \infty\right)}(s) .
$$

- Throughout the paper, $c \geq 0$ will stand for a generic constant depending only on $\alpha, \lambda$, and the kernel $\mu$. Moreover, given $\nu \in(0,1)$, we denote by $c_{\nu} \geq 0$ and $\varepsilon_{\nu} \geq 0$ generic constants depending (besides on $\alpha, \lambda, \mu$ ) only on $\nu$, such that $\varepsilon_{\nu} \rightarrow 0$ as $\nu \rightarrow 0$. 
Then, paralleling some ideas of [1], we introduce the functionals (the first depending on the choice of $s_{*}$ )

$$
\Phi_{1}(t)=-\frac{1}{\kappa} \int_{0}^{\infty} \psi(s)\left\langle\partial_{t} u(t), \eta^{t}(s)\right\rangle d s
$$

and

$$
\Phi_{2}(t)=\left\langle\partial_{t} u(t), u(t)\right\rangle .
$$

Observe that, since $S(t)$ is a contraction semigroup,

$$
\sup _{t \geq 0}\left[\left|\Phi_{1}(t)\right|+\left|\Phi_{2}(t)\right|\right] \leq c .
$$

In the sequel, we shall often make tacit use of the Young, the Hölder, and the Poincaré inequalities.

Lemma 4.1. For any $\nu \in(0,1)$, there exists $s_{*}=s_{*}(\nu) \geq 0$ such that, for all measurable sets $\mathcal{F} \subset \mathbb{R}^{+}$and $\mathcal{A}=\mathbb{R}^{+} \backslash \mathcal{F}$, the corresponding functional $\Phi_{1}(t)$ fulfills the differential inequality

$$
\begin{aligned}
\frac{d}{d t} \Phi_{1} \leq & \left(\kappa \omega \hat{\mu}(\mathcal{F})+2 \kappa \hat{\mu}(\mathcal{F})^{2}+\varepsilon_{\nu}\right)\|u\|_{V}^{2}-(1-\nu)\left\|\partial_{t} u\right\|^{2}+\left(2 \hat{\mu}(\mathcal{F})+\varepsilon_{\nu}\right)\|\xi\|_{\mathcal{M}_{\mathcal{F}}}^{2} \\
& +\omega \int_{\mathcal{F}} \mu(s)\langle u, \xi(s)\rangle_{V} d s+c_{\nu}\|\eta\|_{\mathcal{M}_{\mathcal{A}}}^{2}+c_{\nu}\left(\int_{0}^{\infty}-\mu^{\prime}(s)\|\eta(s)\|_{V}^{2} d s+\mathbb{J}[\eta]\right) .
\end{aligned}
$$

Proof. If $\lim _{s \rightarrow 0} \mu(s)<\infty$, simply set $s_{*}=0$. Otherwise, choose $s_{*} \in\left(0, s_{1}\right)$ (if $\mu$ has no jumps, $\left.s_{*} \in \mathbb{R}^{+}\right)$such that

$$
\int_{0}^{s_{*}} \mu(s) d s \leq \frac{\kappa \nu}{2} .
$$

The time-derivative of $\Phi_{1}$ is given by

$$
\frac{d}{d t} \Phi_{1}=-\frac{1}{\kappa} \int_{0}^{\infty} \psi(s)\left\langle\partial_{t} u, \partial_{t} \eta(s)\right\rangle d s-\frac{1}{\kappa} \int_{0}^{\infty} \psi(s)\left\langle\partial_{t t} u, \eta(s)\right\rangle d s .
$$

We now proceed to the estimate of the two terms in the right-hand side of (4.4).

$\diamond$ The First Term. Using the second equation of system (1.2), we have

$$
\begin{aligned}
& -\frac{1}{\kappa} \int_{0}^{\infty} \psi(s)\left\langle\partial_{t} u, \partial_{t} \eta(s)\right\rangle d s \\
& =-\frac{1}{\kappa}\left\|\partial_{t} u\right\|^{2} \int_{0}^{\infty} \psi(s) d s-\frac{1}{\kappa} \int_{0}^{\infty} \psi(s)\left\langle\partial_{t} u(t), T \eta(s)\right\rangle d s .
\end{aligned}
$$

Since $\psi(s) \leq \mu(s)$ and equality holds for $s \geq s_{*}$, from (4.3) we get

$$
-\frac{1}{\kappa}\left\|\partial_{t} u\right\|^{2} \int_{0}^{\infty} \psi(s) d s \leq-\left(1-\frac{\nu}{2}\right)\left\|\partial_{t} u\right\|^{2} .
$$

Integrating by parts in $s$, and noting that (cf. [12])

$$
\lim _{s \rightarrow 0} \mu(s)^{1 / 4}\|\eta(s)\|=\lim _{s \rightarrow \infty} \mu(s)\|\eta(s)\|=0,
$$


we obtain

$$
\begin{aligned}
-\frac{1}{\kappa} \int_{0}^{\infty} \psi(s)\left\langle\partial_{t} u, T \eta(s)\right\rangle d s & =\frac{1}{\kappa} \int_{0}^{\infty}-\psi^{\prime}(s)\left\langle\partial_{t} u, \eta(s)\right\rangle d s+\frac{1}{\kappa} \sum_{n} \mu_{n}\left\langle\partial_{t} u, \eta\left(s_{n}\right)\right\rangle \\
& \leq \frac{1}{\kappa}\left\|\partial_{t} u\right\|\left(\int_{0}^{\infty}-\psi^{\prime}(s)\|\eta(s)\| d s+\sum_{n} \mu_{n}\left\|\eta\left(s_{n}\right)\right\|\right) .
\end{aligned}
$$

Observe that $\psi$ is continuous in $s_{*}$ and that

$$
\psi^{\prime}(s)=\chi_{\left(s_{*}, \infty\right)}(s) \mu^{\prime}(s) .
$$

Moreover, as $s_{*}<s_{1}$ (if $\mu$ has jumps),

$$
\sum_{n} \mu_{n} \leq \mu\left(s_{*}\right)
$$

Then, we get the estimate

$$
\begin{aligned}
& \int_{0}^{\infty}-\psi^{\prime}(s)\|\eta(s)\| d s+\sum_{n} \mu_{n}\left\|\eta\left(s_{n}\right)\right\| \\
& =\int_{s_{*}}^{\infty}-\mu^{\prime}(s)\|\eta(s)\| d s+\sum_{n} \mu_{n}\left\|\eta\left(s_{n}\right)\right\| \\
& \leq\left(\int_{s_{*}}^{\infty}-\mu^{\prime}(s) d s \int_{s_{*}}^{\infty}-\mu^{\prime}(s)\|\eta(s)\|^{2} d s\right)^{1 / 2}+\left(\sum_{n} \mu_{n} \sum_{n} \mu_{n}\left\|\eta\left(s_{n}\right)\right\|^{2}\right)^{1 / 2} \\
& \leq \frac{\mu\left(s_{*}\right)^{1 / 2}}{\lambda^{1 / 2}}\left[\left(\int_{0}^{\infty}-\mu^{\prime}(s)\|\eta(s)\|_{V}^{2} d s\right)^{1 / 2}+\mathbb{J}[\eta]^{1 / 2}\right] \\
& \leq \frac{\sqrt{2} \mu\left(s_{*}\right)^{1 / 2}}{\lambda^{1 / 2}}\left(\int_{0}^{\infty}-\mu^{\prime}(s)\|\eta(s)\|_{V}^{2} d s+\mathbb{J}[\eta]\right)^{1 / 2} \cdot
\end{aligned}
$$

Therefore,

$$
-\frac{1}{\kappa} \int_{0}^{\infty} \psi(s)\left\langle\partial_{t} u, T \eta(s)\right\rangle d s \leq \frac{\nu}{2}\left\|\partial_{t} u\right\|^{2}+\frac{\mu\left(s_{*}\right)}{\kappa^{2} \lambda \nu}\left(\int_{0}^{\infty}-\mu^{\prime}(s)\|\eta(s)\|_{V}^{2} d s+\mathbb{J}[\eta]\right) .
$$

Collecting the above inequalities yields

$$
\begin{aligned}
& -\frac{1}{\kappa} \int_{0}^{\infty} \psi(s)\left\langle\partial_{t} u, \partial_{t} \eta(s)\right\rangle d s \\
& \leq-(1-\nu)\left\|\partial_{t} u\right\|^{2}+\frac{\mu\left(s_{*}\right)}{\kappa^{2} \lambda \nu}\left(\int_{0}^{\infty}-\mu^{\prime}(s)\|\eta(s)\|_{V}^{2} d s+\mathbb{J}[\eta]\right) .
\end{aligned}
$$

$\diamond$ The Second Term. From the first equation of system (1.2), it holds that

$$
\begin{aligned}
& -\frac{1}{\kappa} \int_{0}^{\infty} \psi(s)\left\langle\partial_{t t} u, \eta(s)\right\rangle d s \\
& =\omega \int_{0}^{\infty} \psi(s)\langle u, \eta(s)\rangle_{V} d s+\frac{1}{\kappa} \int_{0}^{\infty} \psi(s)\left(\int_{0}^{\infty} \mu(\sigma)\langle\eta(s), \eta(\sigma)\rangle_{V} d \sigma\right) d s
\end{aligned}
$$


Let us evaluate the two terms of the above equality separately. Concerning the first one, we have

$$
\begin{aligned}
\omega \int_{0}^{\infty} \psi(s)\langle u, \eta(s)\rangle_{V} d s= & -\omega \int_{0}^{s_{*}}\left[\mu(s)-\mu\left(s^{*}\right)\right]\langle u, \eta(s)\rangle_{V} d s+\omega \int_{\mathcal{A}} \mu(s)\langle u, \eta(s)\rangle_{V} d s \\
& +\omega \int_{\mathcal{F}} \mu(s)\langle u, \xi(s)\rangle_{V} d s+\kappa \omega \hat{\mu}(\mathcal{F})\|u\|_{V}^{2} .
\end{aligned}
$$

Due to (4.3), we have the estimate

$$
\begin{aligned}
& -\omega \int_{0}^{s_{*}}\left[\mu(s)-\mu\left(s^{*}\right)\right]\langle u, \eta(s)\rangle_{V} d s \\
& \leq \omega\|u\|_{V} \int_{0}^{s_{*}} \mu(s)\|\eta(s)\|_{V} d s \\
& \leq \omega\left(\frac{\kappa \nu}{2}\right)^{1 / 2}\|u\|_{V}\|\eta\|_{\mathcal{M}} \\
& \leq \kappa \omega \nu^{1 / 2}\|u\|_{V}^{2}+\frac{\omega \nu^{1 / 2}}{8}\|\eta\|_{\mathcal{M}_{\mathcal{A}}}^{2}+\frac{\omega \nu^{1 / 2}}{8}\|\eta\|_{\mathcal{M}_{\mathcal{F}}}^{2},
\end{aligned}
$$

while

$$
\omega \int_{\mathcal{A}} \mu(s)\langle u, \eta(s)\rangle d s \leq \kappa^{1 / 2} \omega\|u\|_{V}\|\eta\|_{\mathcal{M}_{\mathcal{A}}} \leq \kappa \omega \nu\|u\|_{V}^{2}+\frac{\omega}{4 \nu}\|\eta\|_{\mathcal{M}_{\mathcal{A}}}^{2} .
$$

Thus, we obtain

$$
\begin{aligned}
\omega \int_{0}^{\infty} \psi(s)\langle u, \eta(s)\rangle_{V} d s \leq & \kappa \omega\left(\hat{\mu}(\mathcal{F})+\nu+\nu^{1 / 2}\right)\|u\|_{V}^{2}+\left(\frac{\omega \nu^{1 / 2}}{8}+\frac{\omega}{4 \nu}\right)\|\eta\|_{\mathcal{M}_{\mathcal{A}}}^{2} \\
& +\frac{\omega \nu^{1 / 2}}{8}\|\eta\|_{\mathcal{M}_{\mathcal{F}}}^{2}+\omega \int_{\mathcal{F}} \mu(s)\langle u, \xi(s)\rangle_{V} d s
\end{aligned}
$$

The remaining term is controlled as

$$
\begin{aligned}
& \frac{1}{\kappa} \int_{0}^{\infty} \psi(s)\left(\int_{0}^{\infty} \mu(\sigma)\langle\eta(s), \eta(\sigma)\rangle_{V} d \sigma\right) d s \\
& \leq \frac{1}{\kappa}\left(\int_{\mathcal{A}} \mu(s)\|\eta(s)\|_{V} d s+\int_{\mathcal{F}} \mu(s)\|\eta(s)\|_{V} d s\right)^{2} \\
& \leq\left(\|\eta\|_{\mathcal{M}_{\mathcal{A}}}+\hat{\mu}(\mathcal{F})^{1 / 2}\|\eta\|_{\mathcal{M}_{\mathcal{F}}}\right)^{2} \\
& \leq \frac{1+\nu}{\nu}\|\eta\|_{\mathcal{M}_{\mathcal{A}}}^{2}+(1+\nu) \hat{\mu}(\mathcal{F})\|\eta\|_{\mathcal{M}_{\mathcal{F}}}^{2} .
\end{aligned}
$$

Summarizing, we get

$$
\begin{aligned}
- & \frac{1}{\kappa} \int_{0}^{\infty} \psi(s)\left\langle\partial_{t t} u, \eta(s)\right\rangle d s \\
\leq & \kappa \omega\left(\hat{\mu}(\mathcal{F})+\nu+\nu^{1 / 2}\right)\|u\|_{V}^{2}+\left(\frac{\omega \nu^{1 / 2}}{8}+\frac{\omega}{4 \nu}+\frac{1+\nu}{\nu}\right)\|\eta\|_{\mathcal{M}_{\mathcal{A}}}^{2} \\
& +\left(\hat{\mu}(\mathcal{F})(1+\nu)+\frac{\omega \nu^{1 / 2}}{8}\right)\|\eta\|_{\mathcal{M}_{\mathcal{F}}}^{2}+\omega \int_{\mathcal{F}} \mu(s)\langle u, \xi(s)\rangle_{V} d s .
\end{aligned}
$$


Plugging (4.5)-(4.6) into (4.4) and noting that

$$
\|\eta\|_{\mathcal{M}_{\mathcal{F}}}^{2} \leq 2 \kappa \hat{\mu}(\mathcal{F})\|u\|_{V}^{2}+2\|\xi\|_{\mathcal{M}_{\mathcal{F}}}^{2},
$$

we reach the desired conclusion.

Lemma 4.2. For any $\nu \in(0,1)$ and for all measurable sets $\mathcal{F} \subset \mathbb{R}^{+}$and $\mathcal{A}=\mathbb{R}^{+} \backslash \mathcal{F}$, it holds that

$$
\frac{d}{d t} \Phi_{2} \leq-\left(\kappa \omega+\kappa \hat{\mu}(\mathcal{F})-\varepsilon_{\nu}\right)\|u\|_{V}^{2}+\left\|\partial_{t} u\right\|^{2}-\int_{\mathcal{F}} \mu(s)\langle u, \xi(s)\rangle_{V} d s+c_{\nu}\|\eta\|_{\mathcal{M}_{\mathcal{A}}}^{2} .
$$

Proof. Using the first equation of (1.2), we have the equality

$$
\frac{d}{d t} \Phi_{2}=-\kappa(\omega+\hat{\mu}(\mathcal{F}))\|u\|_{V}^{2}+\left\|\partial_{t} u\right\|^{2}-\int_{\mathcal{F}} \mu(s)\langle u, \xi(s)\rangle_{V} d s-\int_{\mathcal{A}} \mu(s)\langle u, \eta(s)\rangle_{V} d s .
$$

But the last integral can be estimated by

$$
-\int_{\mathcal{A}} \mu(s)\langle u, \eta(s)\rangle_{V} d s \leq \kappa^{1 / 2}\|u\|_{V}\|\eta\|_{\mathcal{M}_{A}} \leq \kappa \nu\|u\|_{V}^{2}+\frac{1}{4 \nu}\|\eta\|_{\mathcal{M}_{\mathcal{A}}}^{2},
$$

so that the claim follows.

Lemma 4.3. Let $\mu$ be a given admissible kernel, and let $\mathcal{F} \subset \mathbb{R}^{+}$be a measurable set. Then, for every $T>0$ and every $\left(u_{0}, v_{0}, \eta_{0}\right) \in \mathcal{H}$, with $\left\|\left(u_{0}, v_{0}, \eta_{0}\right)\right\|_{\mathcal{H}} \leq 1$, it follows that

$$
\int_{0}^{T}\left\|\xi^{t}\right\|_{\mathcal{M}_{\mathcal{F}}}^{2} d t \leq \kappa \hat{\mu}(\mathcal{F}) \int_{0}^{T}\|u(t)\|_{V}^{2} d t+c .
$$

We stress that $c \geq 0$ is independent of $\mathcal{F}, T$, and of the choice of $\left(u_{0}, v_{0}, \eta_{0}\right)$ in the unit ball of $\mathcal{H}$.

Proof. For every $\varepsilon>0$, there exists an open set $\mathcal{O} \supset \mathcal{F}$ such that

$$
\hat{\mu}(\mathcal{O})<\hat{\mu}(\mathcal{F})+\varepsilon .
$$

Then $\mathcal{O}$ is a disjoint union of open intervals $\mathcal{I}_{j} \subset \mathbb{R}^{+}$. Setting

$$
\mathcal{O}_{t}=\mathcal{O} \cap(0, t] \quad \text { and } \quad \mathcal{O}^{t}=\mathcal{O} \cap(t, \infty),
$$

on account of (4.1) we have, for every $t>0$,

$$
\begin{aligned}
\left\|\xi^{t}\right\|_{\mathcal{M}_{\mathcal{F}}}^{2} & \leq \int_{\mathcal{O}_{t}} \mu(s)\left\|\xi^{t}(s)\right\|_{V}^{2} d s+\int_{\mathcal{O}^{t}} \mu(s)\left\|\xi^{t}(s)\right\|_{V}^{2} d s \\
& =\int_{\mathcal{O}_{t}} \mu(s)\|u(t-s)\|_{V}^{2} d s+\int_{\mathcal{O}^{t}} \mu(s)\left\|\eta_{0}(s-t)-u_{0}\right\|_{V}^{2} d s .
\end{aligned}
$$

Recalling that $\left\|S(t)\left(u_{0}, v_{0}, \eta_{0}\right)\right\|_{\mathcal{H}} \leq 1$, in view of (1.4) we obtain

$$
\begin{aligned}
\int_{\mathcal{O}^{t}} \mu(s)\left\|\eta_{0}(s-t)-u_{0}\right\|_{V}^{2} d s & \leq 2 \int_{t}^{\infty} \mu(s)\left\|\eta_{0}(s-t)\right\|_{V}^{2} d s+2 \int_{t}^{\infty} \mu(s) d s \\
& =2 \int_{0}^{\infty} \mu(t+s)\left\|\eta_{0}(s)\right\|_{V}^{2} d s+2 \int_{0}^{\infty} \mu(t+s) d s \\
& \leq 2 C e^{-\delta t}(1+\kappa) .
\end{aligned}
$$


Performing a subsequent integration on $[0, T]$, and since $\varepsilon$ is arbitrarily small, we clearly reach the conclusion if we prove that

$$
\int_{0}^{T}\left(\int_{\mathcal{O}_{t}} \mu(s)\|u(t-s)\|_{V}^{2} d s\right) d t \leq \kappa \hat{\mu}(\mathcal{O}) \int_{0}^{T}\|u(t)\|_{V}^{2} d t .
$$

But

$$
\int_{0}^{T}\left(\int_{\mathcal{O}_{t}} \mu(s)\|u(t-s)\|_{V}^{2} d s\right) d t=\sum_{j} \int_{0}^{T}\left(\int_{\mathcal{I}_{j}} \mu(s) \chi_{(0, t]}(s)\|u(t-s)\|_{V}^{2} d s\right) d t,
$$

and, making a change of variable, it is easy to verify that

$$
\int_{0}^{T}\left(\int_{\mathcal{I}_{j}} \mu(s) \chi_{(0, t]}(s)\|u(t-s)\|_{V}^{2} d s\right) d t \leq \kappa \hat{\mu}\left(\mathcal{I}_{j}\right) \int_{0}^{T}\|u(t)\|_{V}^{2} d t .
$$

Summing over $j$, we complete the proof.

5. A sufficient condition. We first recall a general sufficient condition for exponential stability of contraction semigroups. This is really a particular instance of a famous result due to Datko [6]. For the reader's convenience, we provide a short proof (in this case, much easier than Datko's original argument).

Lemma 5.1. Assume that there exists $c \geq 0$ such that

$$
\int_{0}^{\infty}\|S(t) \zeta\|_{\mathcal{H}}^{2} d t \leq c
$$

for any $\zeta \in \mathcal{D}(\mathbb{L})$ with $\|\zeta\|_{\mathcal{H}} \leq 1$. Then $S(t)$ is exponentially stable, with a decay rate (at least) equal to $(2 c e)^{-1}$.

Proof. By a density argument, it is apparent that the above inequality holds for any $\zeta \in \mathcal{H}$ with $\|\zeta\|_{\mathcal{H}}=1$. Select $\varrho \in(0,1)$. Given $\zeta$ of unit norm, let

$$
t^{*}=\sup \left\{t:\|S(\tau) \zeta\|_{\mathcal{H}}>\varrho, \forall \tau \in[0, t]\right\} .
$$

Then, it is readily seen that $t_{*} \leq c / \varrho^{2}$. Therefore, since $S(t)$ is a contraction semigroup,

$$
\|S(t)\|_{L(\mathcal{H})} \leq \varrho, \quad \forall t \geq \frac{c}{\varrho^{2}} .
$$

Using a standard procedure in the theory of linear semigroup (see, e.g., [17]), we conclude that

$$
\|S(t)\|_{L(\mathcal{H})} \leq \frac{1}{\varrho} e^{-\left(\frac{\rho^{2}}{c} \log \frac{1}{\varrho}\right) t} .
$$

In particular, the best decay rate is $(2 c e)^{-1}$, corresponding to $\varrho=e^{-1 / 2}$.

With regard to our particular semigroup $S(t)$, we can specialize the above lemma in a more convenient form.

Lemma 5.2. Let $\mu$ be a given admissible kernel. Assume that there exists $c \geq 0$ such that

$$
\int_{0}^{\infty}\|u(t)\|_{V}^{2} d t \leq c
$$

for any $\left(u_{0}, v_{0}, \eta_{0}\right) \in \mathcal{D}(\mathbb{L})$ with $\left\|\left(u_{0}, v_{0}, \eta_{0}\right)\right\|_{\mathcal{H}} \leq 1$. Then $S(t)$ is exponentially stable. 
Proof. Exploiting the representation formula (2.1) and the fact that $S(t)$ is contractive, we learn that

$$
\int_{0}^{\infty}\left\|\eta^{t}\right\|_{\mathcal{M}}^{2} d t \leq c
$$

Indeed,

$$
\int_{0}^{\infty}\left\|\eta^{t}\right\|_{\mathcal{M}}^{2} d t \leq 2 \kappa \int_{0}^{\infty}\|u(t)\|_{V}^{2} d t+2 \int_{0}^{\infty}\left\|\xi^{t}\right\|_{\mathcal{M}}^{2} d t
$$

and the claim follows from Lemma 4.3 for $\mathcal{F}=\mathbb{R}^{+}$. In view of Lemma 5.1 , we are done provided that we prove the further estimate

$$
\int_{0}^{\infty}\left\|\partial_{t} u(t)\right\|^{2} d t \leq c
$$

To this end, let us consider the functional $\Phi_{1}(t)$ for $\nu=1 / 2$ and $\mathcal{F}=\emptyset$. Then, from Lemma 4.1, we have

$$
\frac{d}{d t} \Phi_{1} \leq c\|u\|_{V}^{2}-\frac{1}{2}\left\|\partial_{t} u\right\|^{2}+c\|\eta\|_{\mathcal{M}}^{2}+c\left(\int_{0}^{\infty}-\mu^{\prime}(s)\|\eta(s)\|_{V}^{2} d s+\mathbb{J}[\eta]\right) .
$$

Setting

$$
\Psi(t)=c\left\|\left(u(t), \partial_{t} u(t), \eta^{t}\right)\right\|_{\mathcal{H}}^{2}+\Phi_{1}(t),
$$

by virtue of the energy equality (2.2), we conclude that

$$
\frac{d}{d t} \Psi+\frac{1}{2}\left\|\partial_{t} u\right\|^{2} \leq c\|u\|_{V}^{2}+c\|\eta\|_{\mathcal{M}}^{2} .
$$

An integration in $t$ on $(0, \infty)$ together with (4.2) entails the sought inequality.

6. Proof of Theorem 3.3. We will reach our goal by showing that Lemma 5.2 applies. Then let $\left(u_{0}, v_{0}, \eta_{0}\right) \in \mathcal{D}(\mathbb{L})$, with $\left\|\left(u_{0}, v_{0}, \eta_{0}\right)\right\|_{\mathcal{H}} \leq 1$. We shall distinguish two cases.

6.1. The case $\beta=0$. For $\nu \in(0,1), \mathcal{F} \subset \mathbb{R}^{+}$and $\mathcal{A}=\mathbb{R}^{+} \backslash \mathcal{F}$ to be determined later, let us consider the functional

$$
\Phi(t)=\Phi_{1}(t)+(1-\nu) \Phi_{2}(t) .
$$

Combining Lemma 4.1 and Lemma 4.2, we have the differential inequality

$$
\begin{aligned}
\frac{d}{d t} \Phi \leq & -\kappa\left(\omega+(1-\omega) \hat{\mu}(\mathcal{F})-2 \hat{\mu}(\mathcal{F})^{2}-\varepsilon_{\nu}\right)\|u\|_{V}^{2}+\left(2 \hat{\mu}(\mathcal{F})+\varepsilon_{\nu}\right)\|\xi\|_{\mathcal{M}_{\mathcal{F}}}^{2}+c_{\nu}\|\eta\|_{\mathcal{M}_{\mathcal{A}}}^{2} \\
& +(\omega-1+\nu) \int_{\mathcal{F}} \mu(s)\langle u, \xi(s)\rangle_{V} d s+c_{\nu}\left(\int_{0}^{\infty}-\mu^{\prime}(s)\|\eta(s)\|_{V}^{2} d s+\mathbb{J}[\eta]\right) .
\end{aligned}
$$

Hence, using the straightforward estimate

$$
\int_{\mathcal{F}} \mu(s)\langle u, \xi(s)\rangle_{V} d s \leq \frac{1}{2}\left(\kappa \hat{\mu}(\mathcal{F})\|u\|_{V}^{2}+\|\xi\|_{\mathcal{M}_{\mathcal{F}}}^{2}\right),
$$


we end up with

$$
\begin{aligned}
\frac{d}{d t} \Phi \leq & -\kappa\left(\omega+\frac{2(1-\omega)-|1-\omega|}{2} \hat{\mu}(\mathcal{F})-2 \hat{\mu}(\mathcal{F})^{2}-\varepsilon_{\nu}\right)\|u\|_{V}^{2} \\
& +\left(2 \hat{\mu}(\mathcal{F})+\frac{|1-\omega|}{2}+\varepsilon_{\nu}\right)\|\xi\|_{\mathcal{M}_{\mathcal{F}}}^{2} \\
& +c_{\nu}\|\eta\|_{\mathcal{M}_{\mathcal{A}}}^{2}+c_{\nu}\left(\int_{0}^{\infty}-\mu^{\prime}(s)\|\eta(s)\|_{V}^{2} d s+\mathbb{J}[\eta]\right) .
\end{aligned}
$$

For $n \in \mathbb{N}$, consider the sets

$$
\mathcal{A}_{n}=\left\{s \in \mathbb{R}^{+}: n \mu^{\prime}(s)+\mu(s) \leq 0\right\} .
$$

Since

$$
\bigcup_{n} \mathcal{A}_{n}=\mathbb{R}^{+} \backslash\left(\mathcal{F}_{\mu} \cup \mathcal{N}_{\mu}\right),
$$

where $\mathcal{F}_{\mu}$ is given in Definition 3.2 and $\mathcal{N}_{\mu}$ is the nullset where $\mu^{\prime}$ is not defined, and since the sets $\mathcal{A}_{n}$ are increasingly nested, naming

$$
\mathcal{F}_{n}=\mathbb{R}^{+} \backslash \mathcal{A}_{n},
$$

it holds that

$$
\lim _{n \rightarrow \infty} \hat{\mu}\left(\mathcal{F}_{n}\right)=\mathcal{R}_{\mu} .
$$

Choosing $\mathcal{F}=\mathcal{F}_{n}$ and $\mathcal{A}=\mathcal{A}_{n}$ in the estimates for $\Phi(t)$, we introduce the additional functional (depending on $\nu \in(0,1)$ and $n \in \mathbb{N}$ )

$$
\Psi(t)=c_{\nu}(1+n)\left\|\left(u(t), \partial_{t} u(t), \eta^{t}\right)\right\|_{\mathcal{H}}^{2}+\Phi(t),
$$

which, in light of (4.2), fulfills the bound

$$
\sup _{t \geq 0}|\Psi(t)| \leq n c_{\nu}
$$

Note that

$$
\int_{0}^{\infty}-\mu^{\prime}(s)\|\eta(s)\|_{V}^{2} d s+\mathbb{J}[\eta] \geq \int_{\mathcal{A}_{n}}-\mu^{\prime}(s)\|\eta(s)\|_{V}^{2} d s \geq \frac{1}{n}\|\eta\|_{\mathcal{M}_{\mathcal{A}_{n}}}^{2} .
$$

Thus, from (2.2),

$$
\frac{d}{d t}\left(c_{\nu}(1+n)\left\|\left(u, \partial_{t} u, \eta\right)\right\|_{\mathcal{H}}^{2}\right)+c_{\nu}\|\eta\|_{\mathcal{M}_{\mathcal{A}_{n}}}^{2}+c_{\nu}\left(\int_{0}^{\infty}-\mu^{\prime}(s)\|\eta(s)\|_{V}^{2} d s+\mathbb{J}[\eta]\right) \leq 0,
$$

and so we conclude that

$$
\begin{aligned}
\frac{d}{d t} \Psi \leq & -\kappa\left(\omega+\frac{2(1-\omega)-|1-\omega|}{2} \hat{\mu}\left(\mathcal{F}_{n}\right)-2 \hat{\mu}\left(\mathcal{F}_{n}\right)^{2}-\varepsilon_{\nu}\right)\|u\|_{V}^{2} \\
& +\left(2 \hat{\mu}\left(\mathcal{F}_{n}\right)+\frac{|1-\omega|}{2}+\varepsilon_{\nu}\right)\|\xi\|_{\mathcal{M}_{\mathcal{F}_{n}}}^{2} .
\end{aligned}
$$

Integrating this inequality in $t$ on the interval $[0, T]$, with $T>0$ arbitrary, by means of Lemma 4.3 we find the integral estimate

$$
\left(\omega+(1-\omega-|1-\omega|) \hat{\mu}\left(\mathcal{F}_{n}\right)-4 \hat{\mu}\left(\mathcal{F}_{n}\right)^{2}-\varepsilon_{\nu}\right) \int_{0}^{T}\|u(t)\|_{V}^{2} d t \leq n c_{\nu}
$$


Since $\nu \in(0,1)$ can be chosen arbitrarily small, the theorem is proved if we show that, for some $n \in \mathbb{N}$ big enough,

$$
\omega+(1-\omega-|1-\omega|) \hat{\mu}\left(\mathcal{F}_{n}\right)-4 \hat{\mu}\left(\mathcal{F}_{n}\right)^{2}>0 .
$$

This follows from (6.1) and the strict inequality

$$
\omega+(1-\omega-|1-\omega|) \mathcal{R}_{\mu}-4 \mathcal{R}_{\mu}^{2}>0
$$

that is,

$$
\mathcal{R}_{\mu}<M(\omega) .
$$

6.2. The case $\beta>0$. We consider the functional $\Phi_{2}(t)$ for $\mathcal{F}=\mathbb{R}^{+}$and $\nu$ small enough, in order to get from Lemma 4.2 the differential inequality

$$
\begin{aligned}
\frac{d}{d t} \Phi_{2} & \leq-\left(\frac{\kappa \omega}{2}+\kappa\right)\|u\|_{V}^{2}+\left\|\partial_{t} u\right\|^{2}-\int_{0}^{\infty} \mu(s)\langle u, \xi(s)\rangle_{V} d s \\
& \leq-\frac{\kappa}{2}(\omega+1)\|u\|_{V}^{2}+\left\|\partial_{t} u\right\|^{2}+\frac{1}{2}\|\xi\|_{\mathcal{M}}^{2}
\end{aligned}
$$

Then, we define

$$
\Psi(t)=\frac{1}{2 \beta}\left\|\left(u(t), \partial_{t} u(t), \eta^{t}\right)\right\|_{\mathcal{H}}^{2}+\Phi_{2}(t)
$$

Exploiting (2.2), we obtain

$$
\frac{d}{d t} \Psi \leq-\frac{\kappa}{2}(\omega+1)\|u\|_{V}^{2}+\frac{1}{2}\|\xi\|_{\mathcal{M}}^{2}
$$

Now we integrate in $t$ on $[0, T]$, with $T>0$ arbitrary. Applying Lemma 4.3 for $\mathcal{F}=\mathbb{R}^{+}$, we conclude that

$$
\frac{\kappa \omega}{2} \int_{0}^{T}\|u(t)\|_{V}^{2} d t \leq|\Psi(0)|+|\Psi(T)|+c \leq c+\frac{c}{\beta},
$$

due to (4.2).

7. Further applications. In this final section, we mention some possible developments of this theory.

- It is clear that our results also apply to the linear Volterra equation

$$
\partial_{t t} u(t)+\alpha A u(t)+\beta \partial_{t} u(t)-\int_{0}^{t} \mu(s) A u(t-s) d s=0,
$$

which is just a particular case of (1.1), obtained by choosing null past histories. In this case, we do not talk of semigroups anymore, and the exponential stability condition reads

$$
\left\|\left(u(t), \partial_{t} u(t)\right)\right\|_{H \times V} \leq M\left\|\left(u_{0}, v_{0}\right)\right\|_{H \times V} e^{-\varepsilon t}
$$

for some $M \geq 1$ and $\varepsilon>0$. However, it should be noted that the necessary condition (1.4) is used only in the proof of Lemma 4.3, to control the integral

$$
\int_{\mathcal{O}^{t}} \mu(s)\left\|\eta_{0}(s-t)-u_{0}\right\|_{V}^{2} d s
$$


which disappears in the Volterra case. In fact, it is possible to show that Theorem 3.3 holds if we replace (1.4) with

$$
\int_{s}^{\infty} \mu(\sigma) d \sigma \leq K e^{-\vartheta s}
$$

for some $K \geq \kappa$ and $\vartheta>0$. As noted in [1, this is equivalent to requiring that $\mu$ have the exponential decay property; i.e., there exists $\varpi>0$ such that

$$
\int_{0}^{\infty} \mu(s) e^{\varpi s}<\infty
$$

which (at least for sufficiently regular kernels) has been shown to be necessary for exponential decay of solutions [9]. In particular, this Volterra version of Theorem 3.3 extends some results of [15] to more general kernels.

- With the techniques introduced here, we can also establish similar results for the following first-order linear integrodifferential equation, arising in the theory of heat conduction with memory:

$$
\partial_{t} u(t)+\gamma A u(t)+\int_{0}^{\infty} \mu(s) A u(t-s) d s=0,
$$

with $\gamma \geq 0$ and $A, \mu$ as before, supplemented by the initial condition

$$
u(t)=w_{0}(t), \quad t \leq 0,
$$

where $w_{0}$ is an assigned datum. Here, for $\gamma>0$ we have the necessary and sufficient condition (1.4) for exponential stability, while for $\gamma=0$ we find the constraint $\mathcal{R}_{\mu}<1 / 2$. Again, analogous results hold for the Volterra case.

- We finally mention that the results obtained for the linear case can be exported to the nonlinear nonhomogeneous case, providing the existence of global and exponential attractors for the related (nonlinear) semigroup (cf. [3]).

Acknowledgments. I thank Claudio Giorgi for fruitful discussions. I am also indebted to Stefania Gatti for many valuable suggestions.

\section{REFERENCES}

[1] J.A.D. Appleby and D.W. Reynolds, On necessary and sufficient conditions for exponential stability in linear Volterra integro-differential equations, J. Integral Equations Appl. 16 (2004), 221-240. MR.2108285 (2005j:45006)

[2] V.V. Chepyzhov and V. Pata, Some remarks on stability of semigroups arising from linear viscoelasticity, Asymptot. Anal. 46 (2006), 251-273.

[3] M. Conti and V. Pata, Weakly dissipative semilinear equations of viscoelasticity, Commun. Pure Appl. Anal. 4 (2005), 705-720. MR2172716

[4] C.M. Dafermos, Asymptotic stability in viscoelasticity, Arch. Rational Mech. Anal. 37 (1970), 297308. MR0281400 (43:7117)

[5] C.M. Dafermos, Contraction semigroups and trend to equilibrium in continuum mechanics, in "Applications of Methods of Functional Analysis to Problems in Mechanics" (P. Germain and B. Nayroles, Eds.), pp. 295-306, Lecture Notes in Mathematics, no. 503, Springer-Verlag, Berlin-New York, 1976. MR 0521351 (58:25196)

[6] R. Datko, Extending a theorem of A.M. Liapunov to Hilbert space, J. Math. Anal. Appl. 32 (1970), 610-616. MR0268717 (42:3614)

[7] M. Fabrizio and B. Lazzari, On the existence and asymptotic stability of solutions for linear viscoelastic solids, Arch. Rational Mech. Anal. 116 (1991), 139-152. MR.1143437(92k:73040) 
[8] M. Fabrizio and A. Morro, Mathematical problems in linear viscoelasticity, SIAM Studies in Applied Mathematics, no. 12, SIAM, Philadelphia, 1992. MR1153021(93a:73034)

[9] M. Fabrizio and S. Polidoro, Asymptotic decay for some differential systems with fading memory, Appl. Anal. 81 (2002), 1245-1264. MR1956060 (2004a:45015)

[10] C. Giorgi and B. Lazzari, On the stability for linear viscoelastic solids, Quart. Appl. Math. 55 (1997), 659-675. MR1486541(98h:73050)

[11] C. Giorgi, J.E. Muñoz Rivera, and V. Pata, Global attractors for a semilinear hyperbolic equation in viscoelasticity, J. Math. Anal. Appl. 260 (2001), 83-99. MR.1843969 (2002g:35030)

[12] M. Grasselli and V. Pata, Uniform attractors of nonautonomous systems with memory, in "Evolution Equations, Semigroups and Functional Analysis" (A. Lorenzi and B. Ruf, Eds.), pp. 155178, Progr. Nonlinear Differential Equations Appl., no. 50, Birkhäuser, Boston, 2002. MR1944162 $(2003 \mathrm{j}: 37135)$

[13] Z. Liu and S. Zheng, On the exponential stability of linear viscoelasticity and thermoviscoelasticity, Quart. Appl. Math. 54 (1996), 21-31. MR1373836 (97f:73019)

[14] Z. Liu and S. Zheng, Semigroups associated with dissipative systems, Chapman \& Hall/CRC Research Notes in Mathematics, no. 398, Chapman \& Hall/CRC, Boca Raton, FL, 1999. MR1681343 (2000c:47080)

[15] J.E. Muñoz Rivera, Asymptotic behaviour in linear viscoelasticity, Quart. Appl. Math. 52 (1994), 629-648. MR1306041 (95j:73052)

[16] V. Pata and A. Zucchi, Attractors for a damped hyperbolic equation with linear memory, Adv. Math. Sci. Appl. 11 (2001), 505-529. MR1907454 (2003f:35027)

[17] A. Pazy, Semigroups of linear operators and applications to partial differential equations, SpringerVerlag, New York, 1983. MR0710486 (85g:47061) 